\title{
Fire Monitoring, Prevention and Control System for Market Shops
}

\author{
Gachada B. Dubukumah \\ Department of E.E.E. \\ MAUTECH \\ Yola, Nigeria
}

\author{
Uko Victor Sorochi \\ Department of E.E.E. \\ ATBU \\ Bauchi, Nigeria
}

\author{
Abubakar Salisu \\ Department of E.E.E. \\ MAUTECH \\ Yola, Nigeria
}

\begin{abstract}
Supreme likelihoods of fire disasters in buildings from consulted literatures includes: abnormal rise in temperature, gas leakages from gas cylinders and her accessories, and increasing concentration of smoke from burning materials. On this premise, three respective sensors were saddle with the responsibility of monitoring any anomalies from the aforementioned likelihoods of fire in market shops of which their presence above threshold possess a treat to lives and properties. These sensors are: LM35 for monitoring temperature, MQ5 and MQ7 for monitoring gas and smoke respectively. For LM35, a threshold of $50^{\circ} \mathrm{C}$ was used as a threshold for alarm and for MQ5 and MQ7, a threshold of 1000PPM (Part per Million) was used as the alarm threshold. Each sensor was grafted into four respective modeled market stalls. Threshold for smoke and gas were chosen based on the standard set by OSHA (Occupational Safety and Health Administration) and that of temperature was chosen based on the firing point of most materials that can easily kindle in flames, predominantly found in market stalls as discovered in consulted literatures. Once smoke is detected and its concentration approaches threshold, a fire fighting mechanism which is positioned strategically in the market stall, is triggered automatically to combat the fire generating the smoke. A GSM (Global System for Mobile Communication) module called SIM 800L is used to send notification as alarm through text message to owners of respective market shops when threshold is being approached. Lastly, an LCD (Liquid Crystal Display) display is used to display the status of all sensors to users. The brain of the whole project control rest on the shoulders of a microcontroller called ATMEGA328p.
\end{abstract}

\section{General Terms}

Microcontroller based buildings security system

\section{Keywords}

Temperature sensor, gas sensor, microcontroller, siren, fire fighter and encoder

\section{INTRODUCTION}

Fire is a rapid chemical combination of three elements; fuel, heat and oxygen resulting in the production of heat and flame. Automated fire security systems are basically certain form of programmable electronic devices that can be used to identify threats or attacks on buildings. In other to abolish the traditional methods of preventing fire in markets places using human interferences, this project was implemented to design and construct a fire monitoring, prevention and control system for market shops. Any likelihoods of fire disaster in a market shop is intelligently detected using sensors, controlled to avoid viral spread using a fire fighting mechanism and lastly, issuance of a prompt notification using a GSM SMS and a siren or alarm system. Hence, satisfying the needs of the jet age in tackling fire menace in market places

The ATMEGA328p microcontroller was chosen because of the number of its available input/output ports which was sufficient for the project construction without any redundancy, the ease of its programming and the affordability. Fig. 1 gives the block diagram of the construction. The block diagram presents a pictorial explanation of how each component is connected in the physical implementation.

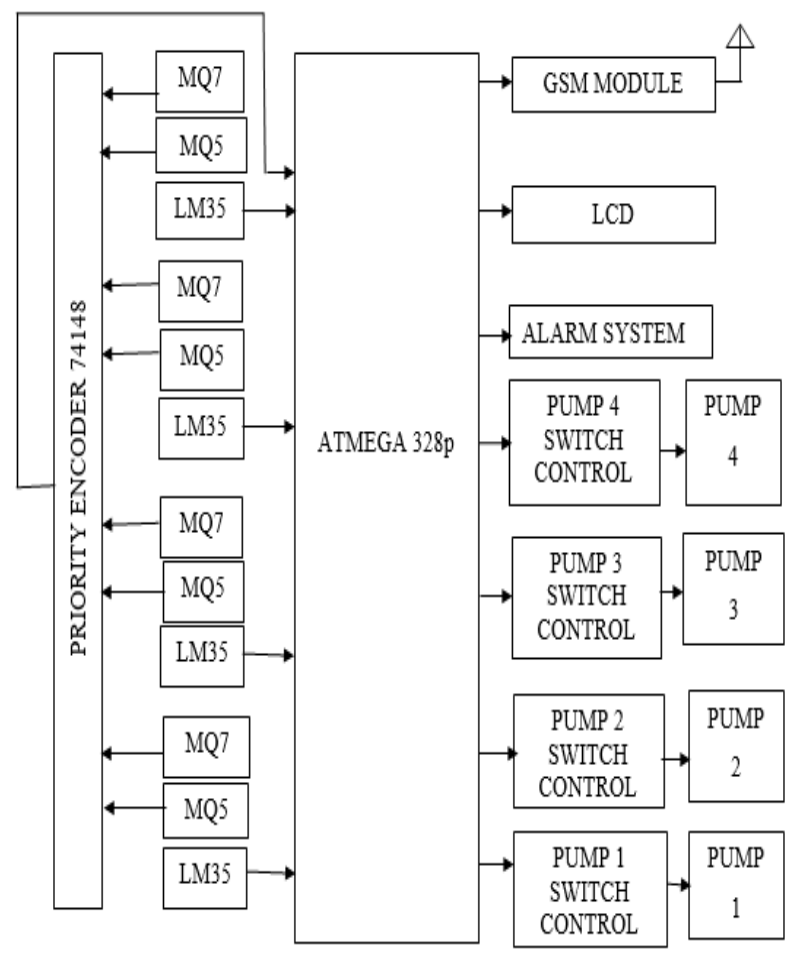

Fig 1: Block diagram of the physical implementation

\subsection{Component Description}

\subsubsection{ATMEGA $328 p$}

This is a high-performance Atmel's 8-bit microcontroller. It is a low-power, high-performance based microcontroller which combines $32 \mathrm{~KB}$ ISP flash memory with read-while-write capabilities, 1KB EEPROM, 2KB SRAM, 23 general purpose I/O lines, 32 general purpose working registers, a single 16-bit Timer/Counter with independent prescalar, compare and capture modes. Other core features of the ATMEGA328 microcontroller are wide operating voltage range: $1.8 \mathrm{~V}$ to 
$5.5 \mathrm{~V}$, maximum operating frequency of $20 \mathrm{MHZ}$, Data retention for 20 years at $85^{\circ} \mathrm{C}$ and 100 years at $25^{\circ} \mathrm{C}$ and 10 bit, 6 channel analog to digital Converter. With these unique features, the ATMEGA328p was preferred for the project construction

\subsubsection{Priority encoder 74148}

A priority encoder is a logical device that accept more than one input on its input line and assigns superiority to each of them at the output. Hence, a priority encoder is a multiple input combination logic circuit. The use of an encoder in this project was necessitated due to the insufficient number of pins on the microcontroller to harbor the output of the eight gas and smoke sensors. The 74148 encoder is an eight input and three output priority encoder. This necessitated its used since its input can harbor the number of smoke and gas sensors to be used without redundancy, moreover, it was chosen because of its affordability and compact sizes. Other types of priority encoders include those with sixteen input and four output such as $\mathrm{MCH} 4536$ which was not suitable for the project because there will be unutilized number of pins.

\subsubsection{Liquid crystal display (LCD)}

Liquid crystal display is an electronic display module that finds a wide range of application in circuits. It is preferred over seven segment display and matrix boards because of its portability, ease to interface with ATMEGA328p and its affordability. A 16 by 2 LCD used in this project means it can display sixteen character per line and there are two of such lines. There are two types of connections made when using the LCD. They include the eight bit mode and the four bit mode. When using the eight bit mode, eleven out of the sixteen pinout of the LCD are used to transfer data per byte while the four bit uses seven out of the sixteen pinout to transfer data per byte. The four bit mode was used in this project as it is much economical than the eight bit mode.

\subsubsection{Sensors (LM 35, MQ7, and MQ5)}

The sensors used for this project were chosen based on the digital and analogue ports available on the microcontroller. For the temperature sensor, an analogue output sensor called LM35 was used since digital port spaces were allocated for encoder pinouts, LCD, siren, pump controllers and GSM module interfacing on the microcontroller. The LM35 was preferred among other analogue temperature sensors such as AD590 and LM36 because of its portability and fast response to temperature changes. The smoke and gas sensors used were MQ7 and MQ5. These sensors were chosen among other type of sensor with digital output because of their simplicity of usage and there fast responses

\subsubsection{GSM module (SIM800L)}

This is a quad-band GSM/GPRS module that works on frequencies GSM850MHz, EGSM900MHz, DSC1800MHz and PSC1900MHz. With a tiny configuration, SIM800L can meet almost all the space requirement in user application such as smart phone, PDA (Personal Digital Assistant) and other mobile device. SIM800L has 88pin pads of LGA (Land Grid Array) packaging and provides all hardware interfaces between the module and users board. It has the following unique features which other GSM module don't have such as programmable general purpose input and output, a SIM card interface, support FM (Frequency Modulation), support PWM (Pulse Width Modulation) and power supply range of $3.4 \mathrm{~V}$ $4.4 \mathrm{~V}$.

\subsubsection{Pump Switching Control}

This unit is saddled with the responsibility of triggering the water pump on or off based on demand. A transistor was used to trigger the relay in the pump switching circuitry after receiving pulses from the microcontroller. A transistor has a fast switching response than any other switching devices like thyristors and triac.

\subsubsection{Pump}

This is a mechanical device constructed for transferring and increasing the flow rate or velocity of water. There are various type of water pumps based on how they are being powered. They include the AC and DC water pump. In this project implementation, a DC water pump was used based on its availability, cost and simplicity of usage and portability.

\subsubsection{Alarm System}

This system shall consist of a loud making device called a siren which will be trigger by the microcontroller when there are any abnormalities in any market shop, detected by any of the grafted sensor based on threshold of calibration.

\section{LITERATURE REVIEW}

\subsubsection{Review of some predecessors' works on the project}

As true as the saying goes that "Rome was not built in a day", lay emphasis on the fact that this project did not just come out of the blues. It rest on building blocks laid by predecessors of similar projects and the principles, theories and discoveries made at different times in history. Reviewing similar projects implemented in the past reveals their level of success, the difficulties encountered, the areas of shortcomings, the recommendation made by the designers which will serve as bedrock for future advancement of the project.

[7] Designed and constructed a fire detector and alarm system for buildings using programmable microcontroller. The basic limitations in the design was the absence of a GSM communication system that can serve as an alternative alerting system through text messages to building owners in cases where the fire alarm is not audible enough to be heard by the building owner due to their distance away from the building. Another limitation was the absence of an automated firefighting mechanism to combat fire accident when it does occurs in a building and lastly, absence of a temperature sensing device for monitoring the building temperature.

In the year (2017), [9] designed and constructed a gas leakage sensing device for residential purposes with GSM feedback mechanism using PIC microcontroller. The project was only limited to gas leakage surveillance as a means of preventing buildings from fire disasters. In addition, there construction was limited to a single structure in a building

[10] Designed a toxic gas release and alarm system using PIC microcontroller system to detect carbon monoxide. The drawbacks of the project was that it was only limited to the detection of only carbon monoxide as the only hazardous gas, inability to relate temperature of the surrounding with toxic gasses released and the inability to have a GSM communication channel as alternative security measure to communicate to her owners when threshold of detected gas is exceeded when they are not at proximity to the implementation to enable them hear the alarm.

In the year (1996), [8] invented a carbon monoxide and safety system. The invention is particularly described in relation to 
measuring carbon monoxide concentration in an automobile garage and for controlling the garage door opener circuit to open the garage door in response to preselected concentration of carbon monoxide. The limitations of the project was the absence of an alarm and GSM system for notification

\subsubsection{Desired Improvement}

Based on the extent of work carried out by project predecessors and by virtue of the limitations in their respective design and implementations, an advancement was made by implementing a low cost integrated fire security and prevention system for market shops which will make use of three different sensors grafted into four individual modeled market shops with some of their outputs channeled to a priority encoder and the output of the encoder going to the microcontroller while others go directly into the microcontroller. Hence, utilizing more ports spaces. The sensors include temperature sensor (LM35) which shall monitor the temperature level of each shop in case of eventualities of fire disaster which is mostly indicated by rising temperature. Smoke sensor (MQ7) which will monitor presence of smoke, giving a direct likelihood of fire disaster and gas sensor MQ5 which shall monitor presence of any gas leakage in the market shop (in cases where gas is used in any market stall). Finally, a firefighting mechanism is incorporated in each of the four markets stalls to fight any fire accident when there is delay in responsiveness of the shop owners or market firefighting agencies to swing into action prior receiving alert from their mobile phones and the shops central siren. Thus, making the system a multisensory based surveillance, mitigation, and prevention system

\section{SYSTEM DESIGN}

The system design is divided into hardware and software design. The hardware design was developed by integrating together the physical components making up the systems block diagram shown in Fig. 1. The software design is aimed at controlling the hardware components of the system. The software design was develop by interpreting the systems flow chat in form of programme codes written in the open-source Arduino environment.

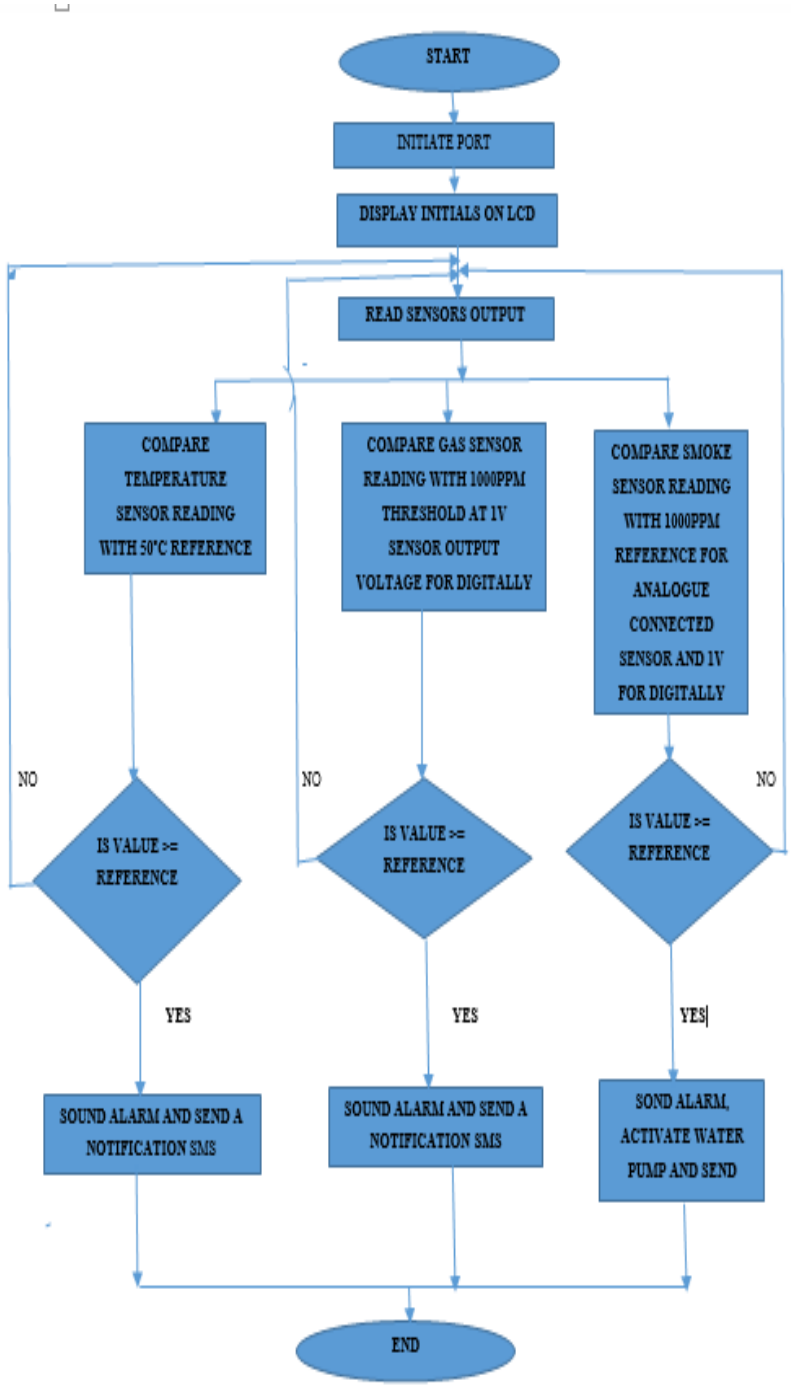

Fig 2: Flow chat for the microcontroller's programming

\section{IMPLEMENTATION AND TESTING}

\subsubsection{Implementation}

In this segment, all paper works that pillared the project design was made handy by physically incorporating the hardware and software component of the design. Initially, the project was implemented on a solderless board to validate its workability and to ease adjustments before the project construction was transferred to a permanent board for soldering. The hardware component incorporation is done in stages based on the system block diagram.

\subsubsection{Testing}

Testing was also done in stages based on the system block diagram to avoid complexity in troubleshooting any abnormality when the whole project reaches completion 


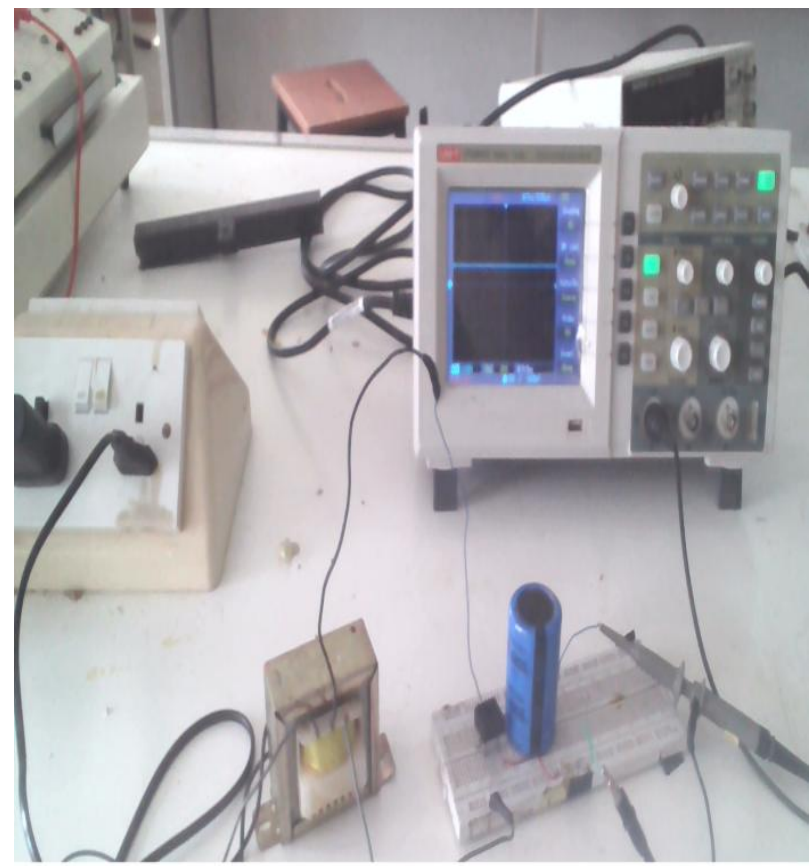

Fig 3: Oscilloscope verification of power supply waveform

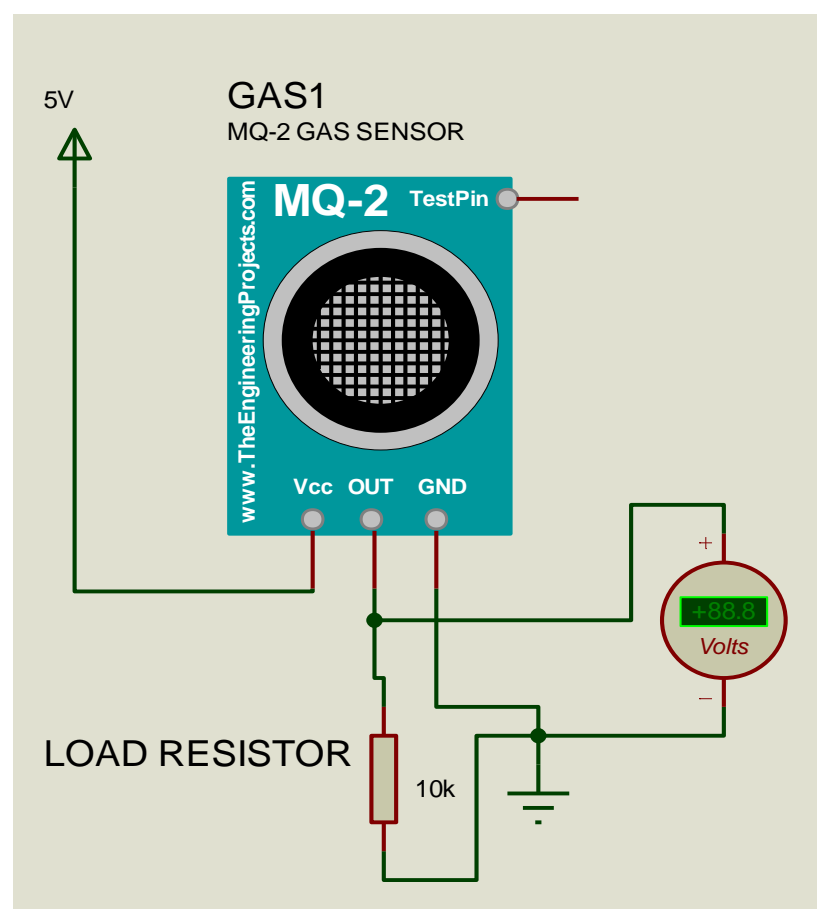

Fig 4: Calibration and test circuit for smoke and gas sensors

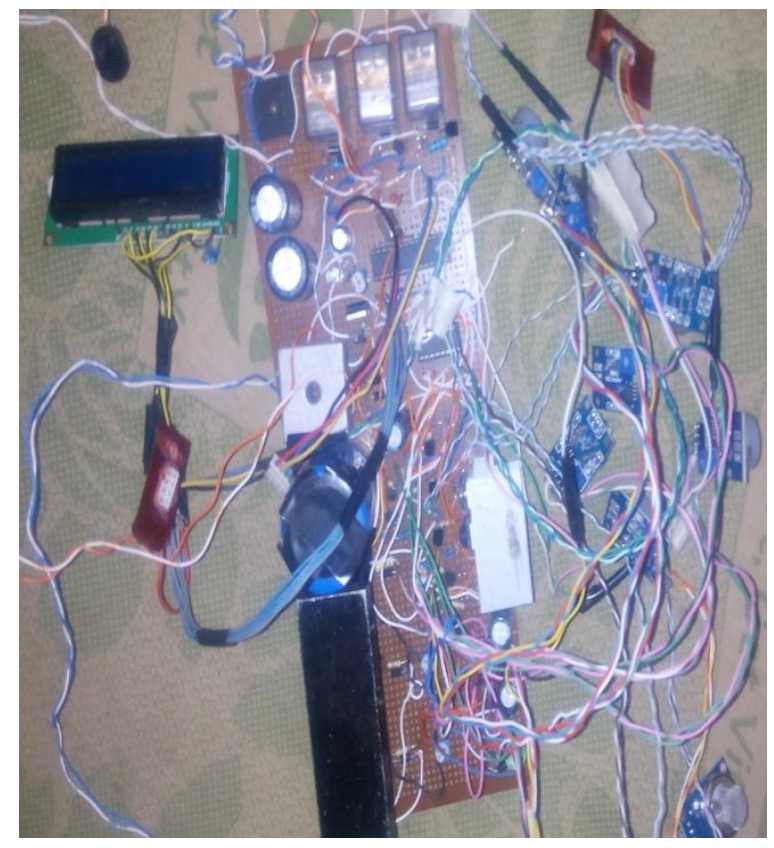

Fig 5: Fully soldered circuit on Vero board

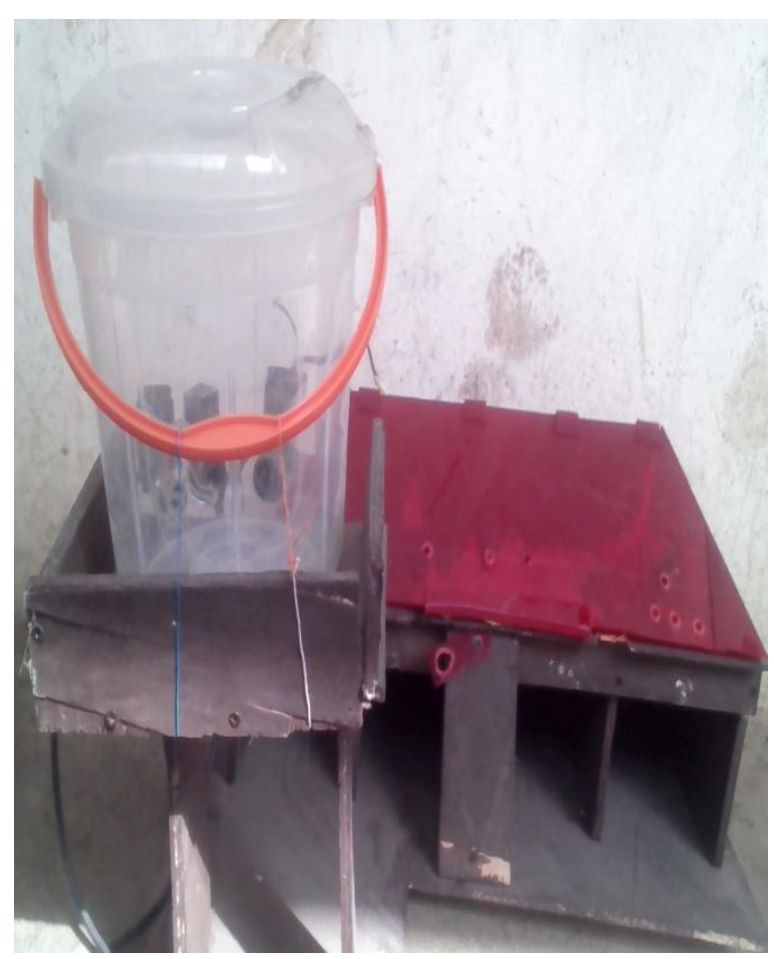

Fig 6: Modeled market shops 
Table 1. Comparison of LCD temperatures with thermometer

COMPARISON TEST OF EACH LCD TEMPERATURE DISPLAY READING WITH STANDARD THERMOMETER TEMPERATURE READING

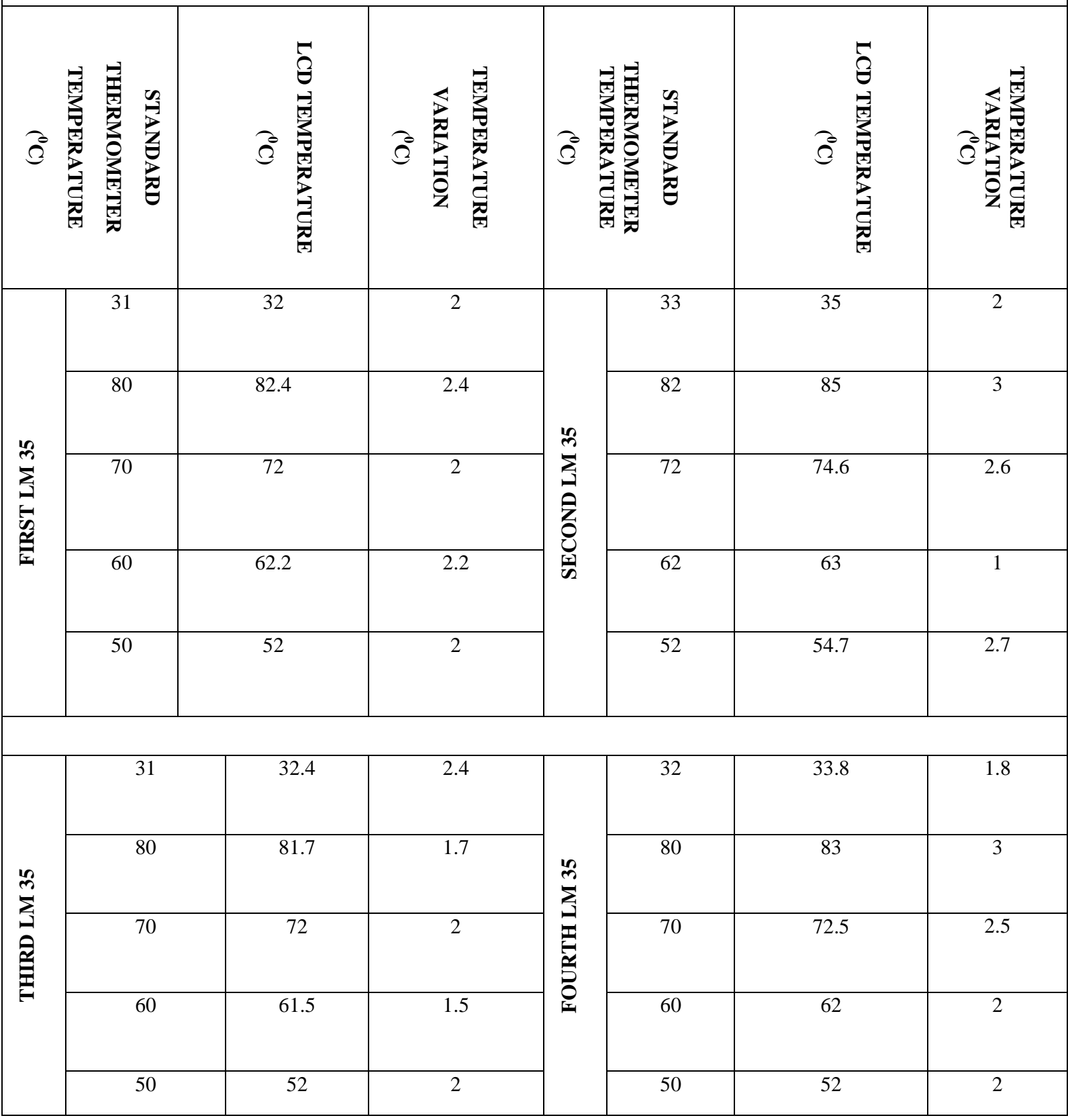




\section{CONCLUSION AND RECOMMENDATION}

\subsubsection{Summary of the work}

From the achievement of the work, the following can be deduced

$>$ The design will find application in houses with physically challenged occupant who are either dumb but learned, as the alarm from the siren and the SMS notification will give insight of an impending danger

$>$ The design will give assurance of safety to building occupant at remote locations to their buildings, when cases of uncertainty arises

\subsubsection{Conclusion}

The aim of the project was achieved based on its ability to monitor, prevent and control and fire accident. With respect to experimental observations, the gas sensors losses there threshold of calibration after a long while. In addition, it was observed that there was an average overshoot of $20 \mathrm{c}$ (as shown in Table 1.) when the temperature reading of the temperature sensors were compared with temperature readings from a standard thermometer subjected under same conditions.

\subsubsection{Recommendations}

The system can be enhanced for future research by incorporating a mechanism that can dispense water from the pump based on smoke levels detected by the smoke sensor. A backup power supply can be included in the system design to augment for power failure conditions. The smoke and gas sensors can be replaced with any better functioning and long lasting sensor such as TGS2442 and MG811. In addition, the design can be improved by incorporating it to the power distribution panel of buildings so that it will be able to automatically trip off power supply to the building when there is any treat. Lastly, the project can be expanded for multiple market shops by using a microcontroller with a higher number of available port spaces such as Atmega32 or by interfacing two microcontrollers together to account for multiple port spaces

\section{ACKNOWLEDGMENTS}

Profound gratitude goes to the Almighty God for His embellished strength and knowledge to the project team while carrying out the research work as an addition to the body of knowledge. The project team can't go forward in appreciations without blowing the trumpet to thank management and staff of Department of Electrical/Electronic Engineering of both Modibbo Adama University of Technology Yola and that of Abubakar Tafawa Balewa University Bauchi for their warm reception and open arms during the project buildups. Needless to mention, this project would not have been completed without reference to the inspirations from the works of predecessors whose details are included in the reference section of this document.

\section{REFERENCES}

[1] Boylestad, R., \& Nashelskyl, P. (1999): Electric devices and circuit theory, $7^{\text {th }}$ Ed New Jersey: Prentice Hall.

[2] Bernard M. O. and John M. C. Electronic Measurements and Implementation, McGraw Hill Book Company, 1983, pp. $712-713$.

[3] Carbon (IV) oxide Safety Limit. (n.d). Retrievedfromhttps://inspectapedia.com/hazmat/carbon\% 20_Dioxide_exposure_limit_php

[4] Defining Alarm Threshold for System Temperature. (n.d.). Retrieved from https://en.m.wikipedia.org/wiki/operating_temperature

[5] Circuit Today. (2015). Fire and GasSystem. Retrieved from http://www.circuitstoday.com/gsm- based Firealarm-system-using Arduino

[6] Green, W. (2008). An Introduction to Indoor Air Quality: Carbon Monoxide (CO). United State Environmental Protection Agency

[7] Khalil, B. S. (2015). Design and Construction of a Fire Detection and Alarm System for Building Using PICMicrocontroller. Abubakar Tafawa Balewa University, Bauchi. (Unpublished)

[8] Murphy, R.F. (1996). Carbon Monoxide Safety System. (United States Patent 5,576,739)

[9] Usman, A. S. and Lawal A. A. (2016). Design and Construction of Gas Leakage Sensing Device for Residential Purpose. Abubakar Tafawa Balewa University, Bauchi. (Unpublished)

[10] Zarith, S. S. (2009). Toxic Gas Release Alarm System Using PIC microcontroller. Universiti Teknologi Malaysia. (Unpublished) 